\title{
IDENTIFICAÇÃO, ESTIMATIVA POPULACIONAL E DESLOCAMENTO DE CARACÓIS EM BORBULHEIRAS DE PLANTAS CÍTRICAS
}

\author{
Eduardo Rodrigues Hickel ${ }^{1}$, Luana Aparecida Castilho Maro² e Marcelo \\ Mendes Haro ${ }^{3}$
}

\begin{abstract}
Resumo: Caracóis têm sido relatados infestando borbulheiras de plantas cítricas em Santa Catarina. Estes moluscos se alimentam das folhas, causando pequenas perfurações no limbo foliar e, principalmente, asco aos trabalhadores rurais. Populações elevadas têm sido observadas, porém sem a real noção das espécies, do número de indivíduos presentes e de sua capacidade de deslocamento no ambiente. Assim o objetivo deste trabalho foi identificar as espécies, estimar a população de caracóis e a velocidade de deslocamento dos indivíduos presentes em borbulheiras de plantas cítricas em ambiente protegido. Um ensaio de marcação e recaptura foi instalado, liberando-se 280 indivíduos marcados de cada espécie, sendo 180 em 18 pontos de liberação para a estimativa populacional e 100, em área central, para mensurar o deslocamento. Os caracóis foram recapturados com armadilhas do solo e por amostragem nas plantas. As espécies de caracóis Bradybaena similaris (Férussac) (Mollusca: Bradybaenidae) e Bulimulus tenuissimus (d'Orbigny) (Mollusca: Bulimulidae) foram identificadas. A população no solo de B. similaris variou de 5,4 a 5,8 indivíduos. $m^{-2}$, enquanto que a de $B$. tenuissimus variou de 2,2 a 7,1 indivíduos. $m^{-2}$. Nas plantas, essas populações foram de 15,0 a 29,6 indivíduos. $m^{-2}$ e de 5,8 a 11,7 indivíduos. $m^{-2}$, respectivamente. Não foi possível mensurar o deslocamento dos caracóis $B$. similaris e $B$. tenuissimus pelo método empregado.
\end{abstract}

Palavras-chave: Demografia. Dispersão. Bradybaena similares. Bulimulus tenuissimus. Mollusca.

\section{Introdução}

Atualmente, as plantas de citros são propagadas vegetativamente por meio de enxertia, o que pode favorecer o acúmulo e disseminação de patógenos, como vírus e bactérias. Devido à latência de alguns destes organismos, os quais podem não apresentar sintomas de infestação por vários anos, é necessário que o material vegetal seja extraído de borbulheiras sadias, manejadas adequadamente (CARVALHO; SILVA; SEMPIONATO, 2000).

Para facilitar a manutenção da sanidade das mudas, é necessária a utilização de cultivo em ambiente protegido. Nesta modalidade de cultivo é possível alterar, de modo acentuado, o ambiente de crescimento e de reprodução das plantas com controle parcial dos efeitos adversos do clima (VIDA et al., 2004). A limitação ao trânsito de espécies de diversos nichos, principalmente inimigos naturais, somado às características climáticas únicas do ambiente protegido, favorecem 0 desenvolvimento de surtos populacionais de espécies oportunistas, que geralmente não são comuns à cultura em questão (LOURENÇO et al., 2002).

Os caracóis se destacam dentre os principais grupos que se aproveitam de alterações ambientais em cultivos agrícolas. Os moluscos são suscetíveis à desidratação, assim, o microclima úmido predominante no ambiente protegido, associado à presença de vegetação ou palhada no solo e a ausência de inimigos naturais, propicia o crescimento populacional destes indivíduos em cultivos de hortaliças, jardins, ambientes controlados, como estufas e casas de vegetação (QUINTELA, 2002).

A infestação de borbulheiras de plantas cítricas por caracóis tem sido frequente em Santa Catarina. Os indivíduos se alimentam das folhas, causando pequenas perfurações no limbo foliar e, principalmente, asco aos trabalhadores rurais. Dependendo do nível de infestação, a perda de área foliar acarreta menor crescimento dos ramos, indispensáveis para a obtenção das borbulhas. Apesar dos relatos referentes ao ataque destes caracóis, pouco se sabe sobre as espécies, o dano e a dispersão dos

\footnotetext{
'E-mail: hickel@epagri.sc.gov.br

²E-mail: luanamaro@epagri.sc.gov.br

${ }^{3}$ E-mail: marceloharo@epagri.sc.gov.br

Epagri - Estação Experimental de Itajaí; Rod. Antônio Heil, 6800; CEP 88318-112, Itajaí, SC, Brasil
} 


\section{REA - Revista de estudos ambientais (Online) v.21, n. 1, p.29-37, jan./jun. 2019}

indivíduos no cultivo.

Assim, o objetivo deste trabalho foi identificar as espécies envolvidas, estimar a população de caracóis presente em borbulheiras de plantas cítricas em ambiente protegido e o deslocamento (distância e velocidade) dos indivíduos nesse ambiente.

\section{Metodologia}

Coleta dos indivíduos: os adultos dos caracóis foram coletados em plantas de citros localizados no ambiente protegido (casa-de-vegetação) utilizado como borbulheira do Programa Fruticultura Tropical da Estação Experimental de Itajaí (EEI) da Empresa de Pesquisa Agropecuária e Extensão Rural de Santa Catarina (Epagri) (2657'06,34"S, 4845'41,33"O, Itajaí-SC).

Identificação das espécies: Indivíduos, representando as principais morfoespécies coletadas nos materiais vegetais, foram acondicionados em frascos contendo álcool $70 \%$ e enviados para o Instituto Oswaldo Cruz, onde foram identificados em nível de espécie.

\section{Bioensaio de marcação e} recaptura: $O$ ambiente protegido tem 1.200 $\mathrm{m}^{2}$ de área e é dividido ao meio por uma calçada cimentada. Apenas metade da área $(16 \times 38 \mathrm{~m})$ foi usada no ensaio. As linhas de plantas estão dispostas perpendicularmente à calçada, em espaçamento de $70 \mathrm{~cm}$ entre linhas e $30 \mathrm{~cm}$ entre plantas, sendo três linhas por cultivar.

Um total de 300 caracóis, de cada espécie identificada, foram recolhidos no ambiente protegido e levados ao laboratório de entomologia da EEI para serem marcados. Apenas indivíduos com tamanho superior a 1 $\mathrm{cm}$ de comprimento foram coletados. Esses caracóis permaneceram $24 \mathrm{~h}$ em aclimatação em caixa plástica $(38 \times 28,5 \times 9 \mathrm{~cm})$, com fundo preenchido por uma camada de $2 \mathrm{~cm}$ de espessura de areia de rio umedecida. Folhas de laranjeira doce (cultivar Lima Verde) foram ofertadas ad libitum como alimento.

No dia seguinte, 180 indivíduos/espécie foram marcados com um pingo de tinta vermelha, na columela da concha, para a medida do deslocamento, e 100 indivíduos/espécie com tinta azul, para a estimativa populacional. Tinta esmalte cosmética de secagem rápida foi utilizada para a marcação. Os caracóis marcados foram levados às borbulheiras, liberando-se os azuis, de ambas as espécies, em 18 pontos de liberação (10 indivíduos de cada espécie por ponto) e os vermelhos, de ambas as espécies, no ponto central da área.

Os pontos de liberação dos caracóis marcados com a cor azul foram estabelecidos em transectos radiais a partir do ponto central da área, sendo seis pontos em cada diagonal da área e mais seis na linha mediana longitudinal. Esses pontos foram distribuídos nas distâncias de 4, 8 e $16 \mathrm{~m}$ a partir do ponto central. Esse procedimento foi adotado visando minimizar 0 efeito da baixa mobilidade destes caracóis, conforme sugerem Parr, Gaskell e George (1968).

Como o solo da casa-de-vegetação é mantido limpo, armadilhas de microclima foram colocadas no solo, constituídas por uma telha francesa de cerâmica sobre o solo, e amostragem nas plantas, usando pano de batida entomológico (1,5×1,0 metros), foram empregadas na recaptura dos caracóis. Os pontos de recaptura foram fixados nos mesmos pontos de liberação dos caracóis marcados de azul, mais oito pontos estabelecidos num raio de $2 \mathrm{~m}$ a partir do ponto central, sendo, aleatoriamente, quatro perpendiculares entre si e quatro nas diagonais da área. As amostragens ocorreram em 14, 17 e 20/10/2016, anotandose o número de caracóis marcados ou não sob as telhas ou presentes numa planta cítrica.

Bioensaios de frequência de captura: Adicionalmente, foram coletados indivíduos sem marcação, nas armadilhas de solo e no pano de batida, com a finalidade de quantificar a abundância absoluta e identificar o hábito das espécies identificadas.

Análises estatísticas: A estimativa populacional de cada espécie foi obtida com as fórmulas de Lincoln (PARR; GASKELL; GEORGE, 1968; SOUTHWOOD, 1987; CHAO; PAN; CHIANG, 2008) (Equação 1) e Bailey (PARR; GASKELL; GEORGE, 1968; SANTOS; FORATTINI, 1999) (Equação 2).

Onde:

$$
\mathrm{N}=\frac{(\mathrm{mxn})}{\mathrm{r}}
$$

$\mathrm{N}$ = Estimativa populacional;

$\mathrm{m}=$ Número de indivíduos marcados liberados no ambiente;

$\mathrm{n}$ = Número de indivíduos coletados na amostragem seguinte a liberação;

$r=$ Número de indivíduos marcados recoletados. 


\section{REA - Revista de estudos ambientais (Online) v.21, n. 1, p.29-37, jan./jun. 2019}

$$
\mathrm{Nc}=\frac{\mathrm{rx}(\mathrm{n}+1)}{(\mathrm{M}+1)}
$$

Onde:

$\mathrm{Nc}=$ Estimativa populacional corrigida;

$r=$ Número de indivíduos marcados liberados; $\mathrm{n}$ =Número de indivíduos coletados na amostragem seguinte;

$\mathrm{m}$ =Número de indivíduos marcados recapturados.

A frequência absoluta de captura, em cada um dos substratos avaliados (solo ou planta), foi analisada empregando-se o teste de frequência Qui-Quadrado $\left(X^{2}\right)$ ao nível de $5 \%$ de probabilidade. Foi verificada a distribuição dos dados e a normalidade com o teste de Shapiro-Wilk, bem como a homogeneidade de variâncias com o teste de Bartlett.

\section{Resultados}

Espécies identificadas: duas espécies de caracóis foram constatadas em borbulheiras de plantas cítricas em ambiente protegido da Epagri, na Estação Experimental de Itajaí:

- Bradybaena similaris (Férussac) (Mollusca: Bradybaenidae): espécie com concha em formato espiral discoidal, apresentando de 1,2 a 1,6 cm de largura na fase adulta (Figura 1A). B. similaris é conhecida popularmente como caracol de jardim, possui origem asiática e está distribuída pela América do Norte, Central e do Sul, onde foi disseminada principalmente pelo comércio de plantas. No Brasil, ocorre de maneira cosmopolita, da região Norte até o Sul (ALMEIDA; BESSA, 2001; JUNQUEIRA et al., 2003; CARVALHO; BESSA; D’ÁVILA, 2008). Este molusco apresenta interesse parasitológico, por ser hospedeiro de Eurytrema coelomaticum Giard et Billet (PINHEIRO; AMATO 1995), Postharmostomum gallinwn Witenberg (DUARTE, 1980) e de Angiostrongylus costaricensis Morera \& Céspedes (RAMBO; AGOSTINI; GRAEFF-TEIXEIRA, 1997).

Essa espécie habita os agroecossistemas hortícolas, podendo eventualmente ser praga de hortaliças folhosas (SOUZA, 1999). B. similaris já foi relatada causando danos em frutíferas como a videira, sendo considerada praga de importância econômica em países asiáticos (CHANG, 1990).

- Bulimulus tenuissimus (d'Orbigny) (Mollusca: Bulimulidae): indivíduos com protoconcha ovalada com cinco a seis voltas, em aspecto cônico, com 1,2 a 2,0 cm de comprimento na fase adulta (Figura 1B) (SILVA et al., 2008). Os moluscos desta espécie apresentam ampla distribuição geográfica, abrangendo áreas da América do Sul e do Norte. No Brasil, é espécie nativa e está presente em todas as regiões do país (SIMONE, 2006). Atua como hospedeiro intermediário de vários parasitos de animais domésticos (THIENGO; AMATO 1995). Esta espécie não é comum causando problemas em ambientes agrícolas abertos, porém, no caso do presente trabalho, em cultivos protegidos em casa-de-vegetação, registrouse pela primeira vez danos causados por esta espécie.

Figura 1 - A) Adulto de B. similaris; B) Adulto de B. tenuissimus (Itajaí - SC, 2018)
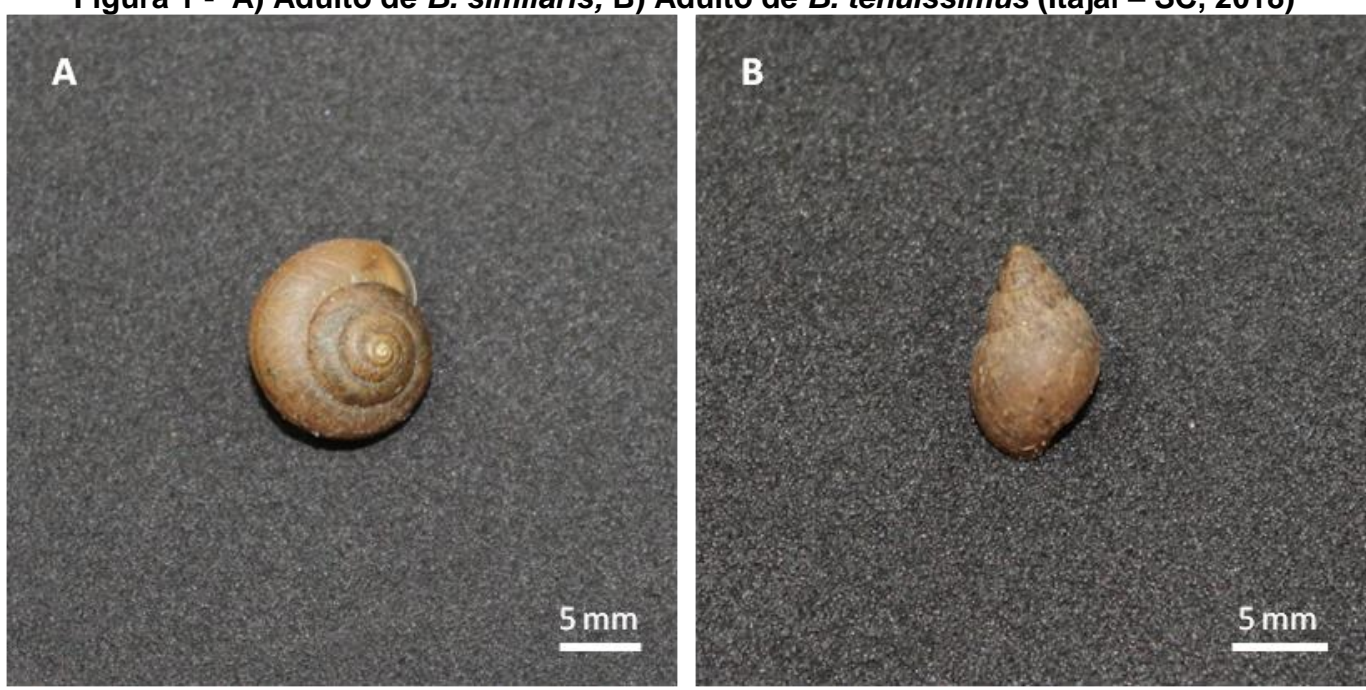

Fonte: Autores (2019) 


\section{REA - Revista de estudos ambientais (Online) v.21, n. 1, p.29-37, jan./jun. 2019}

Danos: Ambas as espécies apresentaram danos semelhantes as plantas de citros, consumindo parte do limbo foliar, de forma irregular, em locais aleatórios, não iniciando, necessariamente, na borda da folha (Figura 2)

Figura 2 - Dano típico do ataque de $B$. similaris e $B$. tenuissimus em folha de citros manejado em cultivo protegido para a retirada de borbulhas (Itajaí-SC, 2018)

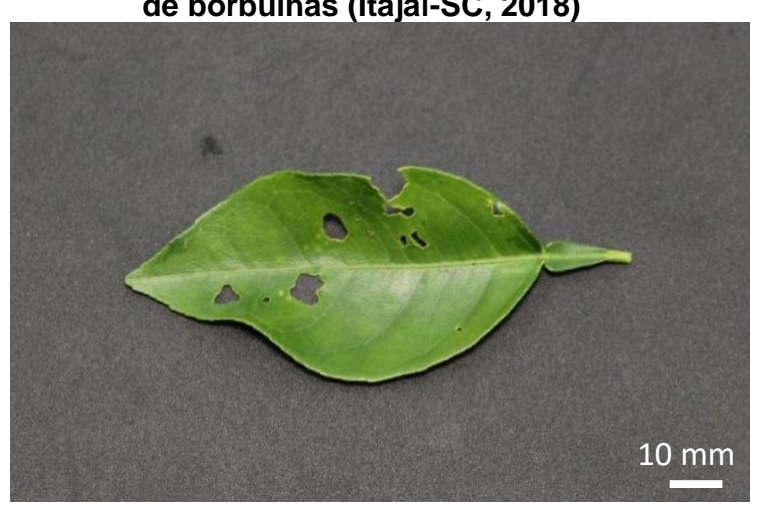

Fonte: Autores (2019)

Frequência de amostragem: $A$ espécie mais abundante e mais destrutiva foi
$B$ similaris, sendo preferencialmente amostrada nas folhas das plantas de citros $\left(X^{2}\right.$ $=11,36 ; p<0,001$ ) (Figura 3). Por outro lado, os indivíduos de $B$. tenuissimus foram amostrados predominantemente no solo $\left(X^{2}=\right.$ 4,$81 ; p=0,028$ ) (Figura 4). No ambiente protegido das borbulheiras, esses caracóis têm encontrado condições propícias para a multiplicação o que, aliado a ausência de predadores nesses ambientes, tem acarretado altas populações.

$B$. similaris está em maior número nas plantas de citros, o que é percebido nas inspeções visuais efetuadas rotineiramente nas borbulheira. Já $B$. tenuissimus é uma espécie de hábitos mais restritos ao solo, onde se alimenta de folhagem em decomposição (MEIRELES et al., 2008; SILVA et al., 2009). Não obstante, os caracóis dessa espécie também sobem nas plantas cítricas, causando os mesmos problemas que a espécie anterior. Segundo Baker (1988a; 1988b) os caracóis tendem a permanecer em maior número nas áreas de forrageamento, onde o deslocamento seria motivado pela escassez de alimento, o que não ocorre neste ambiente.

Figura 3- Frequência de amostragem de $B$. similares no solo e nas plantas de citros. Barras representam o erro padrão da média. (Itajaí -SC, 2018)

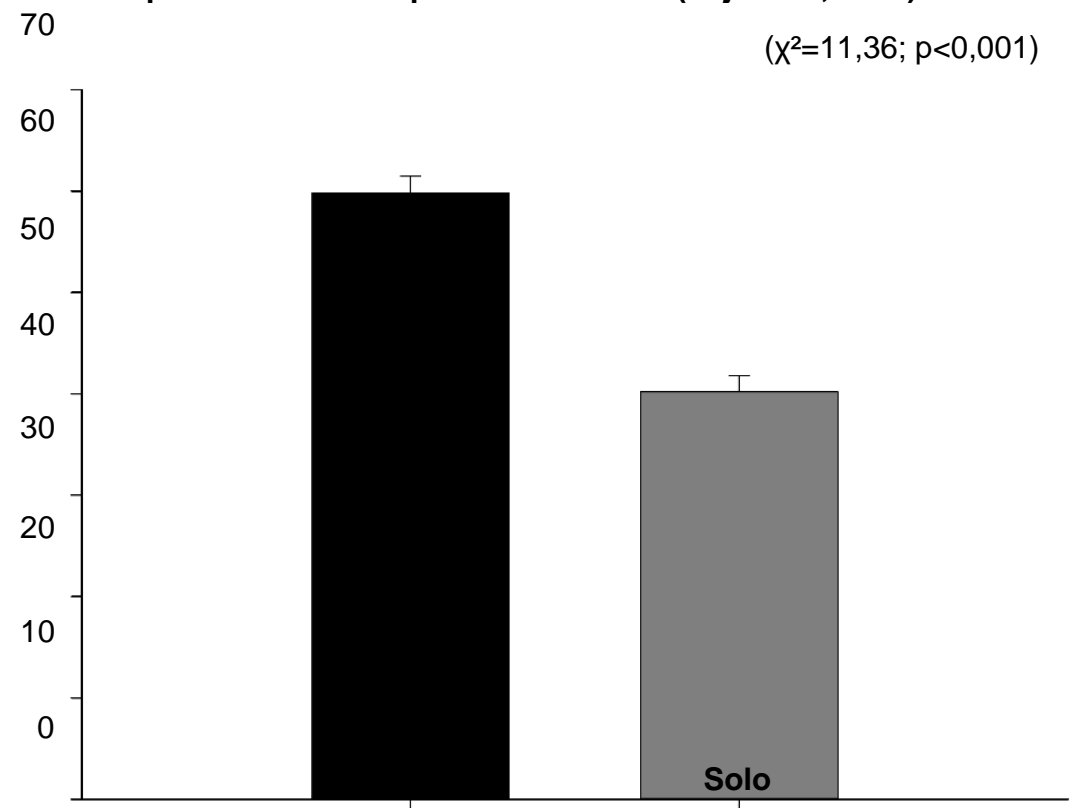




\section{REA - Revista de estudos ambientais (Online) v.21, n. 1, p.29-37, jan./jun. 2019}

Figura 4- Frequência de amostragem de B. tenuissimus no solo e nas plantas de citros. Barras representam o erro padrão da média. (Itajaí-SC, 2018).

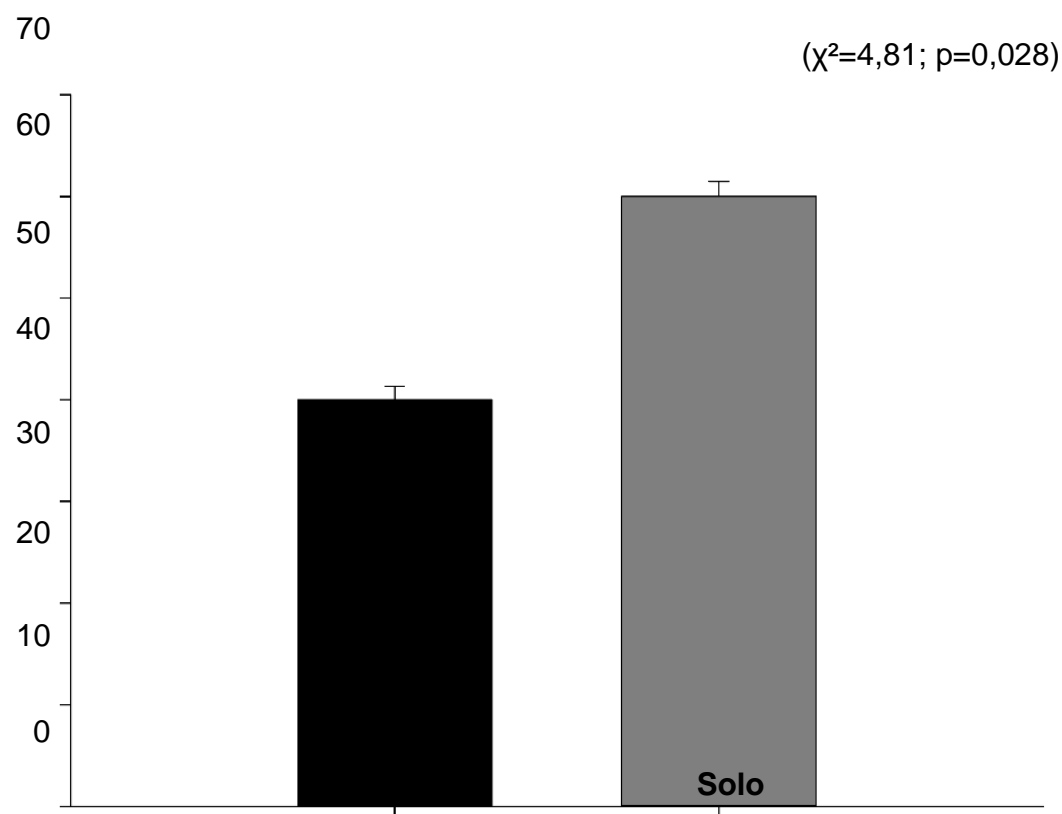

Fonte: Autores (2019)

Bioensaio de marcação e recaptura: A população no solo de $B$. similaris variou de 5,4 a 5,8 indivíduos. $\mathrm{m}^{-2}$, enquanto que a de $B$. tenuissimus variou de 2,2 a 7,1 indivíduos. $\mathrm{m}^{-2}$. Nas plantas essas populações foram maiores, variando de 15,0 a 29,6 indivíduos. $\mathrm{m}^{-2}$ para $B$. similaris e de 5,8 a 11,7 indivíduos. $\mathrm{m}^{-2}$ para $B$. tenuissimus (Tabelas 1 e 2).

O estimador de Lincoln, embora adequado para o caso em estudo, tem o viés de superestimar as populações quando 0 número de marcados recapturados é reduzido (PARR; GASKELL; GEORGE, 1968; SOUTHWOOD, 1987; CHAO; PAN; CHIANG, 2008). Assim, a correção de Bailey (PARR; GASKELL; GEORGE, 1968; SANTOS; FORATTINI, 1999) foi aplicada aos dados (Tabelas 1 e 2).

Muitos estudos de estimativa populacional de moluscos gastrópodes, com a técnica da marcação e recaptura, visam espécies aquáticas de interesse sanitário. 0 caramujo invasor Bellamya chinensis (Gray) (Mollusca: Viviparidae) teve sua população estimada em 5,2 indivíduos. $\mathrm{m}^{-2}$ em um açude no estado americano de Nebraska (CHAINE et al., 2012). A população de Bithynia tentaculata (L.) (Mollusca: Bithyniidae) variou de 740 a 2.300 indivíduos. $\mathrm{m}^{-3}$ nos pequenos lagos de Quebec, no Canadá (MACRAE; LEPITZKI, 1994) e as populações estimadas dos moluscos Bulinus globosus (Morelet) (Planorbidae) e Biomphalaria pfeifferi (Krauss) (Planorbidae) foram de 3,1 e 3,9 indivíduos por metro de margem de rio, respectivamente (WOOLHOUSE, 1988).

Nas três amostragens subsequentes à liberação dos indivíduos marcados, nenhum caracol com tinta vermelha foi encontrado, evidenciando a lenta e eventual movimentação desses organismos (espécies sedentárias). Almeida e Afonso-Neto (2015), estudando o comportamento de $B$. similaris, reportam que os indivíduos têm períodos prolongados de inatividade, principalmente durante o dia. Pyron e Covich (2003) também obtiveram baixa movimentação de caramujos Neritina punctata Quoy et Gaym. Após uma semana de liberação dos indivíduos marcados. O deslocamento desses caramujos foi estimado em 0,08 a $0,17 \mathrm{~cm} . \mathrm{s}^{-1}$ (PYRON; COVICH, 2003). Da mesma forma, o caramujo $B$. tentaculata também se move pouco, na razão de 25 a $50 \mathrm{~cm} \cdot \mathrm{dia}^{-1}$, sendo o deslocamento favorecido pelo aumento da temperatura da água (MACRAE; LEPITZKI, 1994). O caracol $A$. arbustorum se dispersa na razão de 2,6 a 1,1m ao mês (BAUR, 1986). 
v.21, n. 1, p.29-37, jan./jun. 2019

Tabela 1 - População no solo (N.m-2) e correção de Bailey (Nc.m-2) para Bradybaena similaris e Bulimulus tenuissimus em função dos indivíduos marcados recapturados ( $r$ ) e do total capturado ( $n$ ) nas datas de amostragem seguintes. Itajaí, SC, 2017

\begin{tabular}{|c|c|c|c|c|c|c|c|c|}
\hline \multirow{2}{*}{$\begin{array}{c}\text { Data de } \\
\text { amostragem }\end{array}$} & \multicolumn{4}{|c|}{ B. similaris } & \multicolumn{4}{|c|}{ B. tenuissimus } \\
\hline & $\mathbf{r}$ & $\mathbf{n}$ & N.m $\mathrm{m}^{-2}$ & Nc. $m^{-2} \pm$ d.p. ${ }^{1}$ & $\mathbf{r}$ & $\mathbf{n}$ & N.m-2 & Nc. $m^{-2} \pm$ d.p. ${ }^{1}$ \\
\hline $14 / 10$ & 4 & 52 & 5,4 & $4,4 \pm 2,1$ & 6 & 31 & 2,2 & $1,9 \pm 0,7$ \\
\hline $17 / 10$ & 3 & 42 & 5,8 & $4,5 \pm 2,5$ & 0 & 24 & - & 10,4 \\
\hline $20 / 10$ & 0 & 25 & - & 10,8 & 1 & 17 & 7,1 & $3,8 \pm 3,6$ \\
\hline
\end{tabular}

$1 /$ d.p. - desvio padrão

Fonte: Autores (2019)

Tabela 2 - População nas plantas (N.m-2) e correção de Bailey (Nc. $\mathrm{m}^{2}$ ) para Bradybaena similaris e Bulimulus tenuissimus em função dos indivíduos marcados recapturados ( $r$ ) e do total capturado $(n)$ nas datas amostragens seguintes. Itajaí, SC, 2017

\begin{tabular}{|c|c|c|c|c|c|c|c|c|}
\hline \multirow{2}{*}{$\begin{array}{c}\text { Data de } \\
\text { amostragem }\end{array}$} & \multicolumn{4}{|c|}{ B. similaris } & \multicolumn{4}{|c|}{ B. tenuissimus } \\
\hline & $r$ & $\mathbf{n}$ & N.m-2 & Nc. $m^{-2} \pm$ d.p. ${ }^{1}$ & $\mathbf{r}$ & $\mathbf{n}$ & N.m $\mathrm{m}^{-2}$ & Nc. $m^{-2} \pm$ d.p. ${ }^{1}$ \\
\hline $14 / 10$ & 2 & 72 & 15,0 & $10,1 \pm 7,1$ & 1 & 28 & 11,7 & $6,0 \pm 5,9$ \\
\hline $17 / 10$ & 1 & 71 & 29,6 & $15,0 \pm 14,9$ & 1 & 14 & 5,8 & $3,1 \pm 3,0$ \\
\hline $20 / 10$ & 0 & 34 & - & 14,6 & 0 & 6 & - & 2,9 \\
\hline
\end{tabular}

1/d.p. - desvio padrão

Fonte: Autores (2019) 


\title{
REA - Revista de estudos ambientais (Online) v.21, n. 1, p.29-37, jan./jun. 2019
}

Os caracóis B.similaris e $B$. tenuissimus conseguem percorrer distâncias maiores no ambiente das borbulheiras de plantas cítricas, pois indivíduos são encontrados a mais de $2 \mathrm{~m}$ de altura na tela de revestimento e nos canos do sistema de irrigação. Contudo, os dez dias de condução do ensaio não foram suficientes para estes caracóis alcançarem o raio de $2 \mathrm{~m}$ a partir do ponto de liberação central. Baker (1988a; 1988b), estudando os caracóis Theba pisana (Müller) (Mollusca: Helicidae) e Cernuella virgata (da Costa) (Mollusca: Hygromiidae), constatou que as velocidades de deslocamento foram de 0,1 a 1,1 m.dia-1 e de 0,1 a $0,4 \mathrm{~m}$ dia ${ }^{-1}$ respectivamente. Contudo, essas espécies só alcançaram distâncias maiores com o passar do tempo. Alguns indivíduos de $T$. pisana perfizeram $55 \mathrm{~m}$ em um mês (BAKER, 1988b), ao passo que $C$. virgata alcançou somente $25 \mathrm{~m}$ no mesmo período (BAKER, 1988a). Já a espécie
Chondrina avenacea (Bruguière) (Mollusca: Chondrinidae) é muito mais sedentária, deslocando-se os indivíduos a velocidade de 12 cm.dia-1 (BAUR, B.; BAUR, A., 1994; VALENTINE-DARBY et al., 2008).

Conforme argumenta Chlyeh et al. (2003), essa baixa mobilidade de $B$. similaris e $B$. tenuissimus é interessante para fins de manejo das populações. No caso de borbulheiras de plantas cítricas, uma vez efetuado o controle, a reinfestação será lenta devido ao baixo afluxo de indivíduos para as áreas controladas.

\section{Conclusões}

Os caracóis $B$. similaris e $B$. tenuissimus são sedentários no ambiente protegido de borbulheiras de plantas cítricas e suas populações no solo são menores que nas plantas.

\section{Species identification, population estimation and displacement of snails in a citrus bud nursery}

\begin{abstract}
Snails have been reported infesting citrus bud nurseries. These mollusks feed on leaves, causing small perforations in leaflimbs and disgust rural workers. Commonly, high populations have been observed in citrus bud nurseries, however, there is a lack of information about species, colonization, number of individuals present, and their displacement capacity in the environment agroecosystem. Thus, the objective of this work was to identify snails species, estimate the population size of snails and their displacement (distance and speed) in a citrus bud nursery. A mark-recapture assay was installed, releasing 280 marked individuals from each species, 180 released at 18 points for the population estimate and 100 at a central point to measure displacement. The species identified were Bradybaena similaris (Férussac) (Mollusca: Bradybaenidae) and Bulimulus tenuissimus (d'Orbigny) (Mollusca: Bulimulidae). The snails were recaptured with traps in the soil and sampling in the plants. The soil population of $B$. similaris ranged from 5.4 to 5.8 individuals. $m^{-2}$, while $B$. tenuissimus ranged from 2.2 to 7.1 individuals. $\mathrm{m}^{-2}$. In the plants, B. similaris populations registered 15.0 to 29.6 individuals. $\mathrm{m}^{-2}$ and B. tenuissimus ranged from 5.8 to 11.7 individuals. $\mathrm{m}^{-2}$. It was not possible to measure the displacement of the snails $B$. similaris and $B$. tenuissimus by the employed method.
\end{abstract}

Keywords: Demography; Dispersal; Bradybaena similaris; Bulimulus tenuissimus; Mollusca.

\section{Referências}

ALMEIDA, M.N.; AFONSO-NETO, I.S. O gastrópode Bradybaena similaris (Férussac, 1821) (Pulmonata, Xanthonychidae) como modelo experimental para estudo em laboratório. Acta Biomédica Brasileira, v.6, n.2, p.25-41, 2015.

ALMEIDA, M.N.; BESSA, E.C.A. Estudo do crescimento e da reprodução de Bradybaena similaris (Férussac) (Mollusca, Xanthonychidae) em laboratório. Revista Brasileira de Zoologia, v.18, n.4, p.1115-1122, 2001.

BAKER, G.H. The Dispersal of Cernuella virgata
(Mollusca: Helicidae). Australian Journal of Zoology, v.36, n.5, p.513-520, 1988a.

BAKER, G.H. The Dispersal of Theba pisana (Mollusca: Helicidae). Journal of Applied Ecology, v.25, n.3, p.889-900, 1988b.

BAUR, B. Patterns of dispersion, density and dispersal in alpine populations of the land snail Arianta arbustorum (L.) (Helicidae). Holartic Ecology, v.9, n.2, p.117-125, 1986.

BAUR, B.; BAUR, A. Dispersal of the land snail Chondrina avenacea on vertical rock walls. Malacological Review, v.271, n.2, p.53-59, 1994. 
CARVALHO, C.M.; BESSA, E.C.A.; D’ÁVILA, S. Life history strategy of Bradybaena similaris (Fèrussac, 1821) (Mollusca, Pulmonata, Bradybaenidae). Molluscan Research, v. 28, n.3, p.171-174, 2008.

CARVALHO, S. A.; SILVA, J. A. A.; SEMPIONATO, $O$. R. Produção de borbulhas certificadas de citros no estado de São Paulo. Jaboticabal: Unesp, 2000. 331p.

CHAINE, N.M.; ALLEN, C.R.; FRICKE, K.A.; HAAK, D.M.; HELLMAN, M.L.; KILL, R.A.; NEMEC, K.T.; POPE, K.L.; SMEENK, N.A.; STEPHEN, B.J.; UDEN, D.R.; UNSTAD, K.M.; VANDERHAM, A.E. Population estimate of Chinese mystery snail (Bellamya chinensis) in a Nebraska reservoir. Biolnvasions Records, v.1, n.4, p. 238-287, 2012.

CHANG, C. P. Evaluation of chemical and exclusion methods for control of Bradybaena similaris (Férussac) on grapevine in Taiwan. Agriculture Ecosystems Environment, v. 31, p.85-88, 1990.

CHAO, A.; PAN, H.-Y.; CHIANG, S.-C. The Petersen-Lincoln estimator and its extension to estimate the size of a shared population. Biometrical Journal, v.50, n.6, p.957-970, 2008.

CHLYEH, G.; HENRY, P.-Y.; JARNE, P. Spatial and temporal variation of life-history traits documented using capture-mark-recapture methods in the vector snail Bulinus truncatus. Parasitology, v.127, n.3, p.243-251, 2003.

DUARTE, M.J.F. O ciclo evolutivo de Postharmostomum gallinum Witenberg, 1923, no estado do Rio de Janeiro, Brasil (Trematoda, Brachylaemidae). Rev. Brasil. Biol., v.40, n.4, p.793-809, 1980.

JUNQUEIRA, F.J.; D'ÁVILA, S.; BESSA, E.C.A.; PREZOTO, F. Ritmo de atividade de Bradybaena similaris (Férussac, 1821) (Mollusca, Xanthonychidae) de acordo com a idade. Revista de Etologia, v.5, n.1, p.41-46, 2003.

LOURENCSO, I.; RODRIGUES, S.; FIGUEIREDO, E.; GODINHO, M. C.; AMARO, F.; MEXIA, A. The effect of crop protection strategy on pest and beneficials incidence in protected crops. Meded Rijksuniv Gent Fak Landbouwkd Toegep Biol Wet. v. 67, n. 3, p. 563-573, 2002.

MACRAE, M.; LEPITZKI, D.A.W. Population estimation of the snail Bithynia tentaculata (Gastropoda: Prosobranchia) using mark-recapture and the examination of snail movement in pools. Canadian Field-Naturalist, v.108, n.1, p.58-66, 1994.

MEIRELES, L.M.O.; SILVA, L.C.; JUNQUEIRA, F.O.; BESSA, E.C.A. The influence of diet and isolation on growth and survival in the land snail Bulimulustenuissimus (Mollusca: Bulimulidae) in laboratory. Revista Brasileira de Zoologia, v. 25, n.2, p.224-227, 2008.

PARR, M.J.; GASKELL, T.J.; GEORGE, B.J. Capture-recapture methods of estimating animal numbers. Journal of Biological Education, v.2, n.2, p.95-117, 1968.

PINHEIRO, J.; S.B. AMATO. Eurytrema coelomaticum: influence of the infection on the reproduction and nucleic acids contents in the albumen gland and ovotestis of Bradybaena similaris. Mem. Inst. Oswaldo Cruz, v.90, n.5, p.635-638, 1995.

PYRON, M.; COVICH, A.P. Migration patterns, densities, and growth of Neritina punctulata snails in Rio Espiritu Santo and Rio Mameyes, Northeastern Puerto Rico. Caribbean Journal of Science, v.39, n.3, p.338-347, 2003.

QUINTELA, E. D. Manual de identificação dos insetos e outros invertebrados pragas do feijoeiro. Santo Antônio de Goiás: Embrapa Arroz e Feijão, 2002. 51p.

RAMBO, P.R.; AGOSTINI, A.A.; GRAEFFTEIXEIRA, C. Abdominal angioslrongylosis in soulhernence and parasitic burden in molluscs intermediate hosts from eighteen endemic roci. Mem. Inst.Oswaldo Cruz. V.92, n.1, p. 9-14, 1997.

SANTOS, R.C.; FORATTINI, O.P. Marcaçãosoltura-recaptura para determinar o tamanho da população natural de Anopheles albitarsis (Diptera: Culicidae). Revista de Saúde Pública, v.33, n.3, p. 309-313, 1999

SIMONE, L.R.L. Land and freshwater molluscs of Brazil, São Paulo: EGB, 2006, 390p.

SILVA, L.C.; MEIRELES, L.M.O.; JUNQUEIRA, F.O.; BESSA, E.C.A. Development and reproduction in Bulimulus tenuissimus(Mollusca, Bulimulidae) in laboratory. Revista Brasileira de Zoologia, v.25, n.2, p. 220-223, 2008.

SILVA, L.C.; MEIRELES, L.M.O.; JUNQUEIRA, F.O.; BESSA, E.C.A. Influência da umidade do substrato sobre crescimento, produção de ovos e sobrevivência de Bulimulustenuissimus (d'Orbigny, 1835) (Mollusca, Bulimulidae) sob condições de laboratório. Revista Brasileira de Biociências, v.7, n.2, p.144-149, 2009.

SOUTHWOOD, T.R.E. Ecological methods: with particular reference to the study of insects populations. New York: Chapman and Hall, 1987. $524 p$.

SOUZA, J.L. Cultivo orgânico de hortaliças: brócolis, couve-flor e repolho. Viçosa: CPT, 1999. 134p. (CPT. Série Agricultura Orgânica, 228).

THIENGO, S. C.; AMATO, S. B. Phyllocaullis variegatus (Mollusca: Veronicellidae), A new 
intermediare host for Brachylaima sp. (Digenea: Brachylaimatidae). Memórias do Instituto Oswaldo Cruz, v.90, n.5, p.14-18, 1995.

VALENTINE-DARBY, P.L.; DARBY, P.C.; BENNETS, R.E.; KITCHENS, W.M.; PERCIVAL, H.F. The use of mark-recapture to estimate Florida apple snail (Pomacea paludosa Say) density in wetland habitats. Florida Scientist, v.71, n.2, p.115-127, 2008.

VIDA, J. B.; ZAMBOLIN, L.; TESSMANN, D. J.; BRANDÃO FILHO, J. U. T.; VERZIGNASSI, J. R.; CAIXETA, M. P. Manejo de doenças de plantas em cultivo protegido. Fitopatologia Brasileira, v. 29, n. 4, p. 355-372, 2004.

WOOLHOUSE, M.E.J. A mark-recapture method for ecological studies of schistosomiasis vector snail populations. Annals of Tropical Medicine and Parasitology, v.82, n.5, p.485-497, 1988.

\section{Agradecimentos}

Às Dras. Suzete Gomes e Silvana Thiengo do Instituto Oswaldo Cruz pela identificação das espécies de caracóis. À Fundação de Amparo à Pesquisa e Inovação do Estado de Santa Catarina (Fapesc), ao Conselho Nacional de Desenvolvimento Científico e Tecnológico (CNPq) e à Financiadora de Estudos e Projetos (Finep), pelo suporte financeiro ao desenvolvimento da pesquisa. 